\title{
Aspects of the unitarized soft multipomeron approach in DIS and diffraction
}

\author{
M.B. Gay Ducati ${ }^{1}$, E.G. Ferreiro ${ }^{2}$, M.V.T. Machado ${ }^{1}$, C.A. Salgado ${ }^{3}$ \\ 1 Instituto de Física, Universidade Federal do Rio Grande do Sul, Caixa Postal 15051, CEP 91501-970, Porto Alegre, RS, Brazil \\ 2 Depto. de Física de Partículas, Universidade de Santiago de Compostela, 15706 Santiago de Compostela, Spain \\ 3 CERN, 1211 Geneva 23, Switzerland
}

Received: 22 October 2001 / Revised version: 11 January 2002 /

Published online: 5 April 2002 - (C) Springer-Verlag / Società Italiana di Fisica 2002

\begin{abstract}
We study in detail the main features of the unitarized Regge model (CFKS), recently proposed to describe the small- $Q^{2}$ domain. It takes into account a two-component description with two types of unitarized contributions: one is the multiple pomeron exchange contribution, interacting with the large dipole size configurations, and the other one consists of a unitarized dipole cross section, describing the interaction with the small size dipoles. We analyze the ratio between soft and hard pieces as a function of the virtuality, and also compare the resulting dipole cross section to that from the saturation model. Diffraction dissociation is also considered, showing the scaling violations in diffractive DIS and estimating the corresponding logarithmic slope.
\end{abstract}

\section{Introduction}

The study of a new regime of QCD, that of a high density of partons, has drawn much attention in the last years. The key discovery was the observation at HERA of the fast growth of parton densities (mainly gluons) as the energy increases in experiments of deep inelastic scattering. Taking $\sigma^{\text {tot }} \sim s^{\alpha(0)-1}\left(F_{2} \sim x^{-\alpha(0)+1}\right)$, values of $\Delta \equiv \alpha(0)-1$ in the range $0.1-0.5$ have been reported, depending on the virtuality $Q^{2}$ of the photon. However, this steep growth should be tamed, leading to the expected limit given by the Froissart bound $\left(\sigma \lesssim(\log s)^{2}\right.$ as $\left.s \rightarrow \infty\right)$ [1]. This boundary has been derived from very general properties of the $S$-matrix, namely unitarity. A cross section growing as any positive power of $s$ would violate unitarity at asymptotic energies. Thus, theoretically, some kind of saturation of this growing due to unitarity effects is expected [2]. The dynamics of such very dense partonic systems is very interesting and has been studied by many authors both in DIS [3] and in high energy nuclear interactions [4].

The description of the $\gamma^{*} p$ collision in the frame where the proton is at rest is very appropriate to include unitarity corrections. In this frame, the virtual photon $\gamma^{*}$ emitted by the incoming lepton fluctuates into a $q \bar{q}$ pair. This system then suffers multiple interactions with the proton. Such multiple interactions restore unitarity even in the case where it would be violated in a single collision. In the model developed in $[5,6]$, all these corrections have been taken into account, and their strength is constrained by diffractive data. Therefore, the ratio $\sigma^{\text {diff }} / \sigma^{\text {tot }}$ is related to unitarity corrections. This is a common feature to any realization of the Gribov model [7], where the amount of rescatterings is related to diffractive production by means of AGK-cutting rules [8].

In parton language, the increasing number of gluons in a proton as $x \rightarrow 0$ makes gluon fusion very probable. This fusion produces gluons of higher longitudinal momentum, stopping the growth of those with the smallest $x$. In this way unitarity is not violated. Such a procedure was implemented on theoretical grounds from QCD through multiladder exchange using the GLR formalism [3], giving rise to non-linear effects in the standard linear DGLAP approach. The outstanding quantity emerging from the unitarization procedure is the saturation scale $Q_{\mathrm{s}}^{2}(A, x, b)$, setting the region where saturation phenomenon starts to be meaningful. The QCD-inspired phenomenological model [9], for instance, introduces a quite clear identification for this scale: $Q_{\mathrm{s}}^{2}(x) \sim 1 / R_{0}^{2}(x)$. There the saturation radius $R_{0}(x)$, related with the mean transverse distance between partons, is properly extracted from the small- $x$ data from HERA.

In any of the descriptions, the unitarity corrections are given by the non-linear terms, and the phenomenon of saturation is expected when these terms become important. Since the gluons are the partons driving the high energy processes, the signals of the saturation effects should appear in the observables probing the gluonic content of the proton (or the nucleus) [10]. In the nuclear case, the gluon density is $\sim A^{1 / 3}$ higher than in the proton. This makes unitarity corrections more important for nuclei, producing the well-known shadowing of $F_{2}$ [11]. Saturation will thus start at smaller energies in nuclei than in protons. Such a fact is the main reason for the increasing interest 
in the forthcoming $e A$ experiments, where the nucleus will be studied at energies higher than currently available [11].

The open question is if the unitarity corrections have already shown up at present energies and if the saturation has been reached. In particular, at HERA, they should appear in the small- $x$ and small- $Q^{2}$ data [12]. There are several proposals in this direction [9,13], mainly for the case of heavy-ion collisions [14], but a definitive answer is still missing. The main difficulty that we are faced with is the saturation scale $Q_{\mathrm{s}}$, staying in the transition interval of $1-2 \mathrm{GeV}$, which leads to hiding of the effects in more inclusive observables. In this kinematical region the standard QCD perturbative expansion is expected not to be completely reliable. For instance, higher twist terms to the linear approach should be taken into account in such a domain. Moreover, this is the transition region between the soft and hard domains, i.e. the perturbative approaches (including saturation or properly adjusting initial conditions) and the Regge-inspired models are competing, and both frameworks seem to describe the current small- $x$ data.

Bearing in mind that the saturation phenomenon is required in a complete understanding of the high energy reactions, and that a consistent treatment of both inclusive and diffractive processes should be taken into account, in this work we study derivative quantities using the Regge unitarized CFKS model $[5,6]$. In this hybrid model, both soft (multiperipheral pomeron and reggeon exchanges) and hard (dipole picture) contributions are properly unitarized in an eikonal way with triple pomeron interactions also included. This approach describes the transition region and can be used as initial condition for a QCD evolution at high virtualities [15]. The extrapolation to the higher- $Q^{2}$ domain is also performed here, checking the behavior of the model without including QCD evolution. We discuss the similarities and/or connections with the phenomenological saturation model [9], stressing that a QCD evolution is required for a correct description of higher $Q^{2}$ in the inclusive case. For the diffractive case, such a procedure is not formally required, since the nonperturbative sector is dominant in this case. The diffractive structure function is extrapolated to the available larger- $Q^{2}$ range. In particular, the diffractive logarithmic slope, which has been claimed to be a possible new observable to disentangle the dynamics [16,17], is calculated and compared with the result from the saturation model.

\section{The inclusive case}

We start by briefly reviewing the CFKS approach. It interpolates between low and high virtualities $Q^{2}$, which are related to the dipole separation size, $r$, at the target rest frame, considering a two-component model $[5,6]$. Considering the unifying picture of the color dipoles, the separation into a large size (in [6] it is called $L$ ) and a small size (called $S$ in [6]) components of the $q \bar{q}$ pair is made in terms of the transverse distance $r$ between $q$ and $\bar{q}$. The border value, $r_{0}$, is treated as a free parameter - which turns out to be $r_{0} \sim 0.2 \mathrm{fm}$. Hereafter we use the notation soft for the large size configuration and hard for the small size one.

The soft component considers multiple pomeron exchanges (and reggeon $f$ ) implemented in a quasi-eikonal approach [18]. It also includes the resummation of triple pomeron branchings (the so-called fan diagrams). The initial input is a phenomenological pomeron with fixed intercept $\alpha_{\mathrm{P}}(0)=1+\varepsilon_{\mathrm{P}}=1.2$ (further changes are due to absorptive corrections), and an exponential parametrization for the $t$ dependence is considered. In the impact parameter representation, the $b$-space, it looks like (in photoproduction $\left.Q^{2}=0\right)$ :

$$
\chi^{\mathrm{P}}(s, b) \simeq C_{\mathrm{P}} \frac{f_{\mathrm{P}}}{B_{\mathrm{el}}(s)}\left(\frac{s}{s_{0}}\right)^{\varepsilon_{\mathrm{P}}} \exp \left[-b^{2} / B_{\mathrm{el}}(s)\right],
$$

where $B_{\mathrm{el}}(s)$ is the elastic slope, which is parametrized as in the hadronic reactions. The $f_{\mathrm{P}}$ is an effective pomeronproton coupling. In the electroproduction case, the initial input is described in an analogous way:

$$
\begin{aligned}
\chi^{\mathrm{P}}\left(s, b, Q^{2}\right) & \simeq \frac{C_{\mathrm{P}}}{R\left(x, Q^{2}\right)}\left(\frac{Q^{2}}{s_{0}+Q^{2}}\right)^{\varepsilon_{\mathrm{P}}} x^{-\varepsilon_{\mathrm{P}}} \\
& \times \exp \left[-b^{2} / R\left(x, Q^{2}\right)\right],
\end{aligned}
$$

corresponding to the Regge parametrization for the amplitude of the soft pomeron exchange, similar to the DonnachieLandshoff one [19]. The function $R\left(x, Q^{2}\right)$ comes from the exponential assumption about the $t$ dependence and further transformation to the impact parameter representation. We shall remark here that, in the CFKS approach, the authors consider a pomeron fixed intercept of $\alpha_{\mathrm{P}}(0)=1+\varepsilon_{\mathrm{P}}=1.2, \varepsilon_{\mathrm{P}}=0.2$ (a semi-hard value rather than a soft one). The unitarization effects, described by multipomeron exchanges, lead to an effective intercept

$$
\varepsilon_{\text {eff }}=\frac{\mathrm{d} \ln F_{2}\left(x, Q^{2}\right)}{\mathrm{d} \ell n\left(\frac{1}{x}\right)}
$$

that decreases as $Q^{2}$ or $x$ decrease due to the increase of shadowing effects.

The resummation of the triple pomeron branches is encoded in the denominator of the amplitude $\chi^{n \mathrm{P}}$, i.e. the Born term in the eikonal expansion. Moreover, the corrected amplitude is eikonalized in the total cross section,

$$
\begin{aligned}
\chi^{n \mathrm{P}}\left(x, Q^{2}, b\right) & =\frac{\chi^{\mathrm{P}}\left(x, Q^{2}, b\right)}{1+a \chi_{3}\left(x, Q^{2}, b\right)}, \\
\sigma^{n \mathrm{P}}\left(x, Q^{2}, b\right) & \simeq 1-\exp \left[-\chi^{n \mathrm{P}}\left(x, Q^{2}, b\right)\right],
\end{aligned}
$$

where the constant $a$ depends on the proton-pomeron and the triple pomeron couplings at zero momentum transfer $(t=0)$. References [5,6] give a more detailed discussion.

The eikonalization procedure modifies the growth of the total cross section from a steep power-like behavior to a milder logarithmic increase. The above parametrization corresponds to the interaction with the large size dipole configurations and therefore dominates in low- $Q^{2}$ 
values. The total soft contribution is obtained by integrating over the impact parameter the cross section at fixed $b, \sigma^{n \mathrm{P}}\left(x, Q^{2}, b\right)$,

$$
\sigma^{\mathrm{soft}}\left(s, Q^{2}\right)=4 \int \mathrm{d}^{2} b \sigma^{\mathrm{soft}}\left(s, Q^{2}, b\right) .
$$

The hard component is considered in the color dipole picture of DIS [20]. The dipole cross section, modeling the interaction between the $q \bar{q}$ pair and the proton, $\sigma^{\text {dipole }}$ $(x, r)$, is taken from the eikonalization of the expression above $\chi^{n \mathrm{P}}\left(s, b, Q^{2}\right)$ already corrected by triple pomeron branching (the fan diagrams contributions). The configurations considered are those with a small transverse distance between the quark-antiquark pair in the dipole. The corresponding cross section is extracted by considering the contributions coming from distances between 0 and $r_{0}=0.2 \mathrm{fm}\left(1 \mathrm{GeV}^{-1}\right)$ - see discussion above -, whereas for $r>r_{0}$ the contributions are described by the soft piece already discussed. In such small distances, perturbative QCD is expected to work. The total cross section considering this dipole cross section is expressed as [6]:

$$
\begin{aligned}
\sigma_{\text {tot }}^{\text {hard }}\left(x, Q^{2}\right) & =\int_{0}^{r_{0}} \mathrm{~d}^{2} r \int_{0}^{1} \mathrm{~d} \alpha\left|\Psi_{\gamma^{*} q}^{\mathrm{T}, \mathrm{L}}(\alpha, r)\right|^{2} \sigma_{\mathrm{CFKS}}^{\text {dipole }}(x, r), \\
\sigma_{\text {CFKS }}^{\text {dipole }}(x, r) & =4 \int \mathrm{d}^{2} b \sigma^{n \mathrm{P}}\left(x, Q^{2}, b, r\right), \\
\sigma^{n \mathrm{P}}\left(x, Q^{2}, b, r\right) & \simeq 1-\exp \left[-r^{2} \chi^{n \mathrm{P}}\left(x, Q^{2}, b\right)\right],
\end{aligned}
$$

where $\mathrm{T}$ and $\mathrm{L}$ correspond to transverse and longitudinal polarizations of a virtual photon; $\Psi_{\gamma^{*} q}^{\mathrm{T}, \mathrm{L}}(\alpha, r)$ are the corresponding wave functions of the $q \bar{q}$ pair.

The $r^{2}$ dependence is introduced in the Born term of the eikonal expansion, presented in the last expression above, in order to ensure the correct behavior determined by the color transparency: for small $r$ the growth in radius should be proportional to $r^{2}, \sigma^{n \mathrm{P}}\left(x, Q^{2}, b, r\right) \simeq$ $r^{2} f\left(x, Q^{2}, b\right)$. This condition, valid for fixed $s$ and $Q^{2}$ as $r \rightarrow 0$, is a property of the single pomeron exchange. Thus a factor $r^{2}$ has been introduced in the eikonal of (8).

Another difference between the soft and hard component is the fact that the contribution of the $f$-exchange (reggeon exchanges) to the hard component is very small, and has been neglected [5].

The weight of each contribution (soft and hard) in the total cross section [and $F_{2}\left(x, Q^{2}\right)$ ] can be obtained, providing an analysis of the role played by each piece of the model. Such a procedure allows us to explicit the regions of $x$ and $Q^{2}$ where the sectors contribute. In Figs. 1 and 2 we calculate the ratio $R_{\mathrm{SOFT}}$, defining the fraction of the total contribution arising from the soft sector:

$$
R_{\mathrm{SOFT}}\left(x, Q^{2}\right)=\frac{\sigma_{\mathrm{tot}}^{\mathrm{soft}}\left(x, Q^{2}\right)}{\left[\sigma_{\mathrm{tot}}^{\mathrm{soft}}\left(x, Q^{2}\right)+\sigma_{\mathrm{tot}}^{\mathrm{hard}}\left(x, Q^{2}\right)\right]} .
$$

From Fig. 1 we note that the soft contribution slowly increases as the momentum fraction $x$ goes to higher values, almost independently of the virtuality $Q^{2}$. This is due

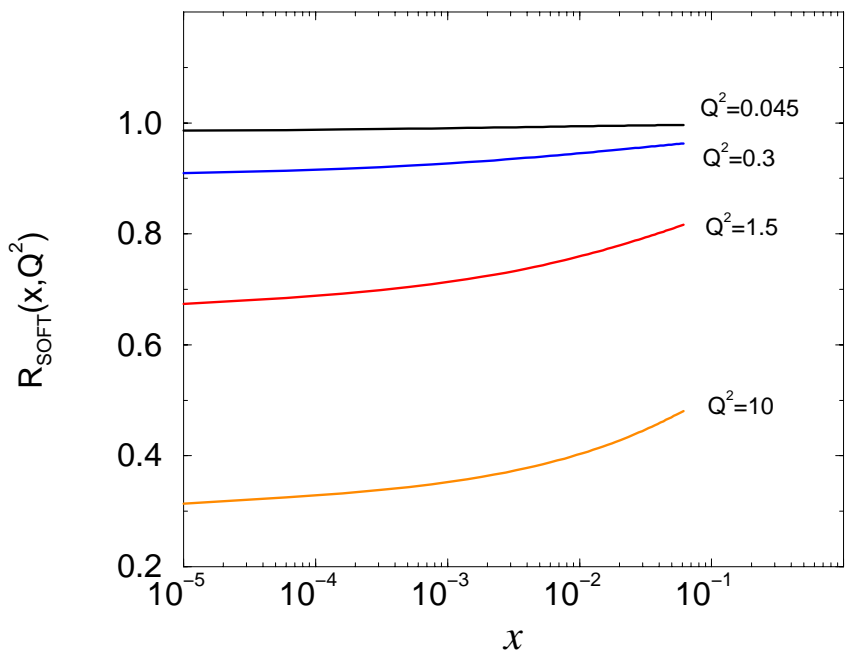

Fig. 1. The ratio $R_{\mathrm{SOFT}}$ as a function of $x$ at fixed virtualities

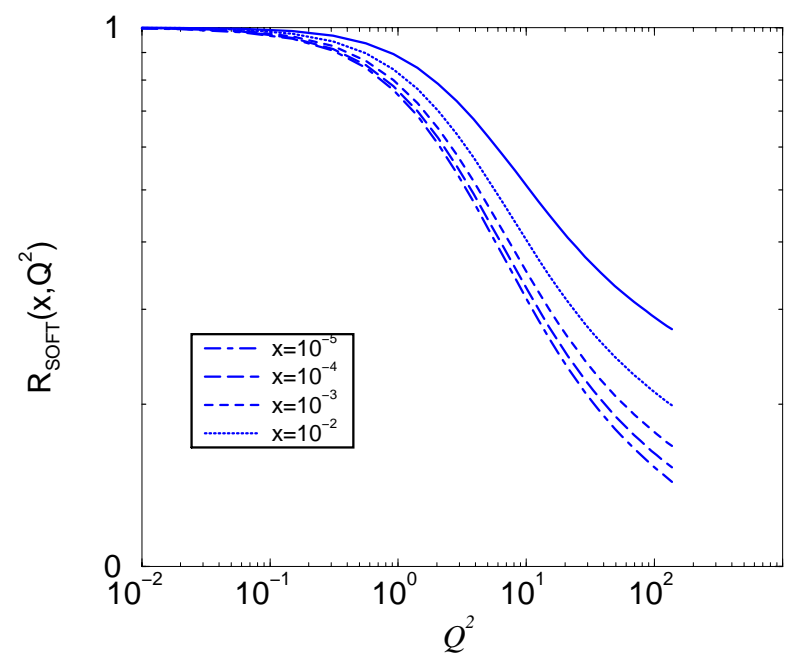

Fig. 2. The ratio $R_{\mathrm{SOFT}}$ as a function of $Q^{2}$ at fixed momentum fraction $x$

to the fact that higher reggeon trajectories $f$ are included in the soft part, but not in the hard one. Regarding fixed virtualities, the soft piece dominates completely the total cross section at $Q^{2}=0.045$. As $Q^{2}$ increases the contribution goes down. For instance, at $Q^{2}=10 \mathrm{GeV}^{2}$ it contributes about half of the cross section. Extrapolating up to higher virtualities, the soft piece saturates at about $5-15 \%$ of the total result.

Figure 2 clearly shows that the soft piece is dominant at $Q^{2}=0.01$ and decreases as the virtuality grows. The behavior is monotonic, almost independent of the momentum fraction $x$. For instance, at $Q^{2}=100 \mathrm{GeV}^{2}$, it contributes with $20 \%$ at $x=10^{-2}$ and $5 \%$ at $x=10^{-5}$. Such a reduction on the soft content is related to the coupling of the photon to the asymmetric dipoles $g_{\text {soft }}^{2}\left(Q^{2}\right) \sim$ $1 /\left(1+Q^{2} / m_{\text {soft }}^{2}\right)$ and to the enhancement in $Q^{2}$ provided by the photon wave function (at high $Q^{2} \gg Q_{\mathrm{s}}^{2}(x)$ the symmetric dipole configuration provides the scaling with logarithmic violation). 
An interesting issue is the relation between the dipole cross section coming from the CFKS model and the phenomenological one of Golec-Biernat-Wüsthoff [9]. The GBW cross section is parametrized by

$$
\begin{aligned}
\sigma^{\mathrm{GBW}}(x, r) & =\sigma_{0}\left[1-\exp \left(-r^{2} / 4 R_{0}^{2}(x)\right)\right], \\
R_{0}^{2}(x) & =\left(\frac{x}{x_{0}}\right)^{\lambda} \mathrm{GeV}^{-2},
\end{aligned}
$$

where $\sigma_{0}=23.03 \mathrm{mb}$ properly normalizes the dipole cross section. The remaining parameters are $\lambda=0.288$ and $x_{0}=3.04 \times 10^{-4}$, all of them determined from the small- $x$ HERA data. The $R_{0}(x)$ is the main theoretical contribution, defining the saturation scale, which is related with the taming of the gluon distribution at small $x$ (unitarity effects) [3]. The above expression has been used to describe both inclusive and diffractive structure functions, in good agreement with the experimental results. The comparison between this approach and the CFKS dipole cross section is shown in Fig. 3. We have plotted the nondimensional result, since the normalization for the CFKS dipole cross section, $\sigma_{0}$, is not determined from data. Indeed, for a comparison with experiment using only the hard piece from CFKS, the adjustable parameters would have to be refitted. We consider here that this can be absorbed by a suitable normalization, and carry the $r$ interval beyond the range set by the model $\left(r<r_{0}\right)$. The main feature of the GBW parametrization is that it ensures that the dipole cross section grows linearly with $r^{2}$ at small transverse separation, whereas it saturates at large size configurations. The picture emerging from the CFKS is slightly different, presenting a mild (logarithmic) increase with $r$, away from huge separation sizes that shift the saturation scale up to very high virtualities. Although continuously and smoothly increasing with the radius, in the CFKS approach the cross section underestimates the GBW one for all $r$.

A comment on the normalization is in order. The GBW formula would correspond to the hard part of CFKS without triple pomeron $(a=0)$, and taking a step function for the profile instead of a gaussian. This makes unitarity corrections stronger. In any case, we can compare GBW and the hard part of CFKS: taking $a=0$ and $\exp \left[-b^{2} /\right.$ $\left.R\left(x, Q^{2}\right)\right] \longrightarrow \Theta\left[b^{2}-R\left(x, Q^{2}\right)\right]$ in CFKS, doing the integral in $b$ and comparing with GBW, one obtains $\sigma_{\text {CFKS }}^{\text {dipole }}$ $(x, r)=\sigma_{0}\left[1-\exp \left(-r^{2} \chi^{\mathrm{P}}\left(s, Q^{2}\right)\right]\right.$, with $\sigma_{0}=\pi R(x$, $\left.Q^{2}\right) \sim 20 \mathrm{mb}$, in agreement with GBW value. This value, however, depends logarithmically on $x$ and $Q^{2}$, because of the increase of the proton radius, which is taken into account in (2). The comparison between the exponents (the eikonals, which contain most of the parameters in CFKS case) of the above expression and of (10) is less clear, as the $x$-dependences are different and the triple pomeron cannot be neglected in this case.

\section{The diffractive case}

The diffractive sector in the CFKS approach is constructed by a three-component model $[5,6]$, using the AGK-cutting

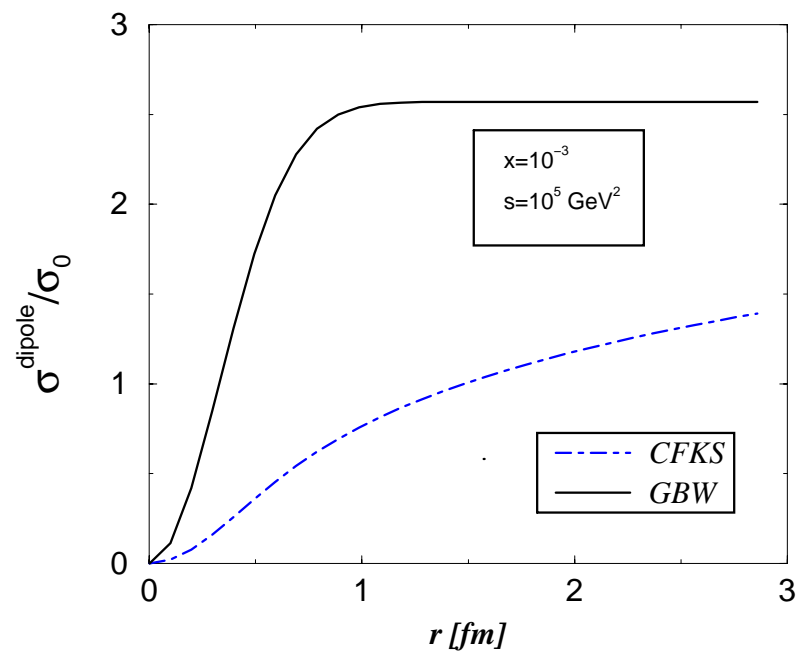

Fig. 3. The comparison between the saturation dipole cross section from Golec-Biernat-Wüsthoff (GBW) and CFKS as a function of the transverse dipole separation $r$ at fixed $x(s)$

rules to relate the elastic multiple scattering amplitude to the inelastic diffractive contribution [8]. The first term comes directly from the soft piece, the second one from the triple pomeron (and the reggeon $f$ ) interaction and the last one from the hard (dipole) piece. We notice that these contributions define only the energy, $s$ (and momentum fraction $x$ ), and the virtuality dependences. The spectrum on $\beta$ is introduced by hand, based on earlier soft and hard (pQCD) calculations. The first component is written as

$$
\begin{aligned}
F_{2(\text { soft })}^{D}\left(x, Q^{2}, \beta\right) & \sim F_{\text {soft }}^{D(\text { Born })} K_{\mathrm{L}}\left(s, Q^{2}\right) \\
& \times \beta^{-\varepsilon_{\mathrm{P}}}(1-\beta)^{n_{p}\left(Q^{2}\right)},
\end{aligned}
$$

where $F^{D}(\text { Born })_{\text {soft }} \sim \chi^{n i}\left(s, Q^{2}\right) \chi^{n k}\left(s, Q^{2}\right)$ is the lowest order (Born) approximation for that function, with $i, k=\mathrm{P}, f$. The suppression factor due to higher order multipomeron exchanges is $K_{\mathrm{L}}\left(s, Q^{2}\right)=\sigma_{\text {soft }}^{(0)} / \sigma_{\text {soft }}^{(0) \text { Born }}$, with $\sigma_{\text {soft }}^{(0)}=4 g_{\mathrm{L}}^{2}\left(Q^{2}\right) \int \mathrm{d}^{2} b\left[\sigma_{\text {soft }}\left(s, Q^{2}, b\right)\right]^{2}$. Further details can be found in [6]. The $\beta$ dependence is taken from the typical CKMT pomeron structure function, which is connected with the deuteron structure function by the identification $x \rightarrow \beta$ [21].

The hard contribution is expressed by

$$
\begin{aligned}
F_{2(\text { hard })}^{D}\left(x, Q^{2}, \beta\right) & \sim \sigma_{\text {hard }}^{(0) \mathrm{L}} \beta^{3}(1-2 \beta)^{2} \\
& +\sigma_{\text {hard }}^{(0) \mathrm{T}} \beta^{2}(1-\beta),
\end{aligned}
$$

where the $\beta$ dependence comes from a pQCD guess for the pomeron structure function [22]. Also, $\sigma_{\text {hard }}^{(0) \mathrm{L}}=\int \mathrm{d}^{2} b$ $\left[\sigma_{\text {hard }}^{\mathrm{T}, \mathrm{L}}\left(s, Q^{2}, b\right)\right]^{2}$. Notice that (13) contains the higher twist terms of the parametrization [22]. The leading twist component would be described in this approach by the $L$ component [6] (named soft here).

Regarding the $\beta$ dependence, the region for medium values $(\beta \sim 0.4)$ is dominated by the soft term, which 


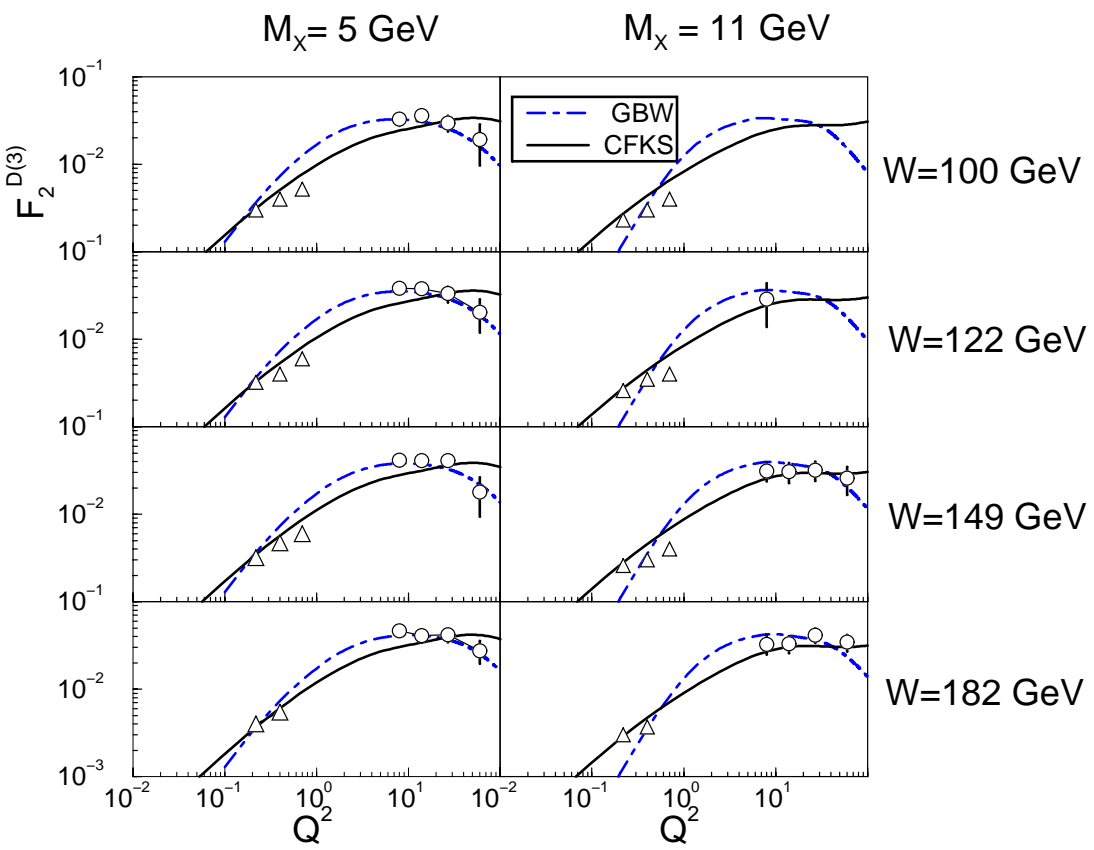

Fig. 4. The diffractive structure function as a function of $Q^{2}$ at fixed $M_{X}$ and $W$. The preliminary data are from ZEUS Collaboration (triangles). The published data are the circles. The CFKS and GBW results are shown in the same plot in pQCD is associated to the transverse photon contribution [22]. The small $\beta$ region is dominated by the triple pomeron piece, in agreement with the pQCD expectations, which is obtained by considering the higher twist $q \bar{q}+$ gluon configuration. Moreover, the hard contribution is leading in the large- $\beta$ region, associated in this case with a suppression of the transverse contribution and an enhancement of the longitudinal piece in comparison with the expected pQCD behavior [22].

The CFKS approach describes with good agreement the diffractive DIS data in the broad range $0<Q^{2}<$ $18 \mathrm{GeV}^{2}$. In order to study the model in comparison with the pQCD approaches, here we extrapolate the prediction for the diffractive structure function of the CFKS approach for higher values of the virtuality. We use the preliminary ZEUS analyses, considering the $Q^{2}$ dependence at fixed mass $M_{X}$ and center-of-mass energy $W$ [23]. These data provide information at both small and large virtualities bins. It is interesting to compare the predictions of the CFKS model and the saturation model [9] for the diffractive structure function. Both models are depicted in the plots of Fig. 4.

The agreement of the CFKS approach with the data is remarkable even at high virtualities, where the model is expected not to be reliable. However the interpretations at low $Q^{2}$ are quite different. In the saturation model, the reliability of the pQCD calculation is extended to smaller virtualities through the saturation scale $R_{0}(x)$, where the dependence is mostly due to the longitudinal photon configuration, by the higher twist $q \bar{q}+$ gluon. Instead, in the CFKS model the main contribution in the region of interest comes from the soft triple pomeron contribution.

As a final study, we perform the calculation of the $Q^{2}$ logarithmic slope of the diffractive structure function $F_{2}^{D(3)}$. The motivation is that this observable is a potential quantity to distinguish soft and hard dynamics in diffractive DIS $[16,17]$. The measurements of the derivative quantity of the $F_{2}$-slope on $Q^{2}$ have lead to renewed interest in testing the matching of hard and soft approaches and have provided constraints for the saturation formalisms. The reported turnover on the $x$ dependence has been associated with the transition region between the soft and hard domains. When we focus on diffractive DIS, in particular the structure function $F_{2}^{D}$, the situation is far from clear: initially considered as a predominantly soft process, the experimental results suggest that the diffractive cross section at HERA contains hard and soft components.

Still, diffraction stands as a more profitable field to study saturation effects than the inclusive case. This comes from the fact that, in DDIS, the large dipole size configuration (soft content) is more relevant than in the DIS reaction [9]. Although there are quite different approaches, based on different physical dynamics, applied to the interpretation of the diffractive measurements, almost all of them fit the data set properly [24]. Therefore a derivative quantity, the diffractive logarithmic slope, has been proposed. It can help to distinguish the underlying dynamics in diffractive DIS, settling the validity range of the different approaches, if such an observable is measured. Here, we have calculated the slope as a function of the pomeron momentum fraction $x_{\mathrm{P}}$ and we have performed a comparison between the CFKS and the GolecBiernat-Wüsthoff (GBW) approaches.

In this calculation, the main feature of the GBW approach is the presence of positive and negative slopes, contrary to the pQCD non-saturated case [17]. In the pQCD model without saturation, the parameters fixed by the previously available data lead to a predominantly positive slope for the whole kinematical region, even at large $Q^{2}$ (large $\beta$ ). We notice that this situation can be changed by a further analysis, considering the updated measurements 

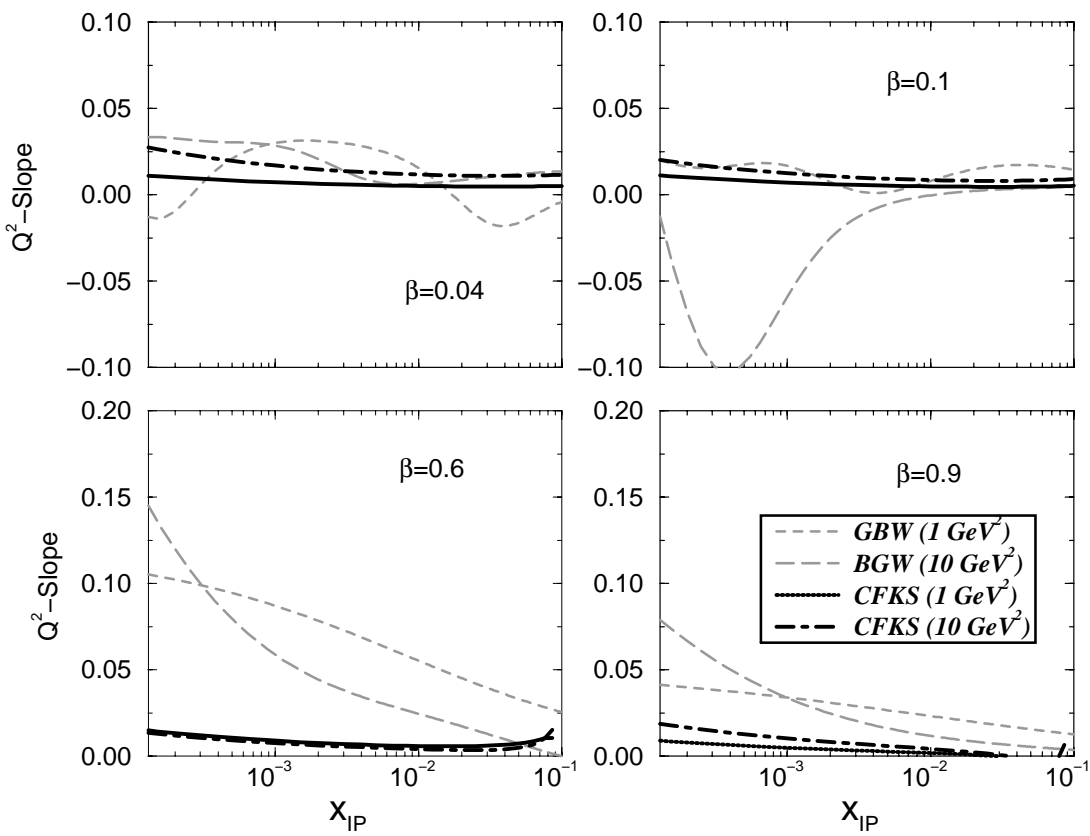

Fig. 5. The $Q^{2}$ logarithmic slope of the diffractive structure function as a function of $x_{\mathrm{P}}$ at fixed $\beta$, for $Q^{2}=1$ and $Q^{2}=10 \mathrm{GeV}^{2}$. The CFKS and GBW results are shown in the same plot of H1 [24], which have enlarged the available kinematical range and have provided new measurements for the regions previously covered.

It is important to emphasize that a transition in the slope takes place in the preliminary ZEUS analyses of diffractive DIS [23] at large virtualities, where the saturation model [9] is considered to describe the $Q^{2}$ dependence of the diffractive structure function. In this model, the analysis is performed using $M_{X}$ and $W$ as kinematical variables instead of $\beta$ and $x_{\mathrm{P}}$, due to the similarity of the behaviors of $\mathrm{d} \sigma / \mathrm{d} M_{X}$ and $\sigma_{\text {tot }}\left(\gamma^{*} p\right)$ in the same kinematical range. There, the growth of $x_{\mathrm{P}} F_{2}^{D}$ versus $Q^{2}$ is stopped at $Q^{2} \sim 10 \mathrm{GeV}^{2}$ and decreases smoothly for larger virtualities. The transition region corresponds to $\beta \sim 0.2$ for $M_{X}=5 \mathrm{GeV}$ and $\beta \sim 0.07$ for $M_{X}=11 \mathrm{GeV}$ [17], $\beta=Q^{2} /\left(Q^{2}+M_{X}^{2}\right)$. These features can be verified observing the plots of Fig. 4 at large virtualities.

Here, we compared the results from the saturation model with those from the model analyzed in this work. They are shown in Fig. 5, where the slope is calculated as a function of $x_{\mathrm{P}}$ for fixed $\beta$ and at two different virtualities. We choose $Q^{2}=1$ and $10 \mathrm{GeV}^{2}$ because this is the region where the CFKS model is formally valid. However, we emphasize that it can be extended to higher virtualities in the diffractive case, since the soft component is stronger than in the inclusive case. The saturation model produces a transition between positive and negative slope values at low $\beta=0.04$ (upper plots), while it shows a positive slope for medium and large $\beta$. Instead, the CFKS approach presents a positive slope for the whole $Q^{2}$ and $x_{\mathrm{P}}$ ranges, flattening at large $\beta$, similarly as the non-saturated $\mathrm{pQCD}$ calculations. These features can be probed if the slope is measured and could help the understanding of the underlying dynamics. Finally, the results above, mainly the ones for the $F_{2}^{D}$ structure function, corroborate the CFKS model as a consistent hybrid approach to describe diffractive DIS, with a close connection to the above mentioned pQCD calculations.

\section{Conclusions}

A deeper understanding of the saturation phenomenon is required to perform reliable estimations for the current and forthcoming high energy reactions. The saturation scale, which sets the onset of the unitarity corrections, is found to be in the transition regime of low $x$ and $Q^{2}$. In this domain, both Regge-inspired phenomenology and improved $\mathrm{pQCD}$ calculations (perturbative shadowing, higher twist), considering unitarity effects, are able to describe the data with high precision. The most advantageous ones are those describing in an unified way the inclusive processes as well as diffractive ones. In this letter we have considered the two-component multireggeon model of $[5,6]$ and calculated some related quantities.

The ratio of the soft content in the model has been calculated, verifying that it dominates at low $Q^{2}$, diminishing at higher virtualities. This shows that the unitarity corrections in this model are more important in the soft component than in the hard one. Moreover, these corrections are higher twist at large $Q^{2}$ in the second case.

We have also studied the robustness of the CFKS model to describe a large range in $Q^{2}$ without considering a pQCD evolution. A good hint to answer this question comes from the analysis of the hard piece (symmetric dipole configurations), in particular the corresponding dipole cross section. We have found that the $r$-saturation of this quantity lies at higher values of the radius than in the phenomenological GBW model. A similar consideration is far from clear for the diffractive case, where the non-perturbative (soft) component plays a more important role. 
We have extrapolated the estimations for the diffractive structure function at high virtualities, verifying that a broad description is obtained, and that it is in reasonable agreement with the saturation pQCD model. An additional quantity has been proposed in order to describe the dynamics of the diffractive dissociation [16,17], in particular the diffractive slope. It has been calculated using the CFKS model, and its main feature is a behavior similar to the one predicted by pQCD calculations [22].

Acknowledgements. M.B.G.D and M.V.T.M are supported by CNPq and by PRONEX (Programa de Apoio a Núcleos de Excelência), Brazil. E.G.F is supported by the contract AEN990589-C02 of CICYT of Spain. C.A.S. is supported by a Marie Curie Fellowship of the European Community program TMR (Training and Mobility of Researchers), under the contract number HPMF-CT-2000-01025.

\section{References}

1. M. Froissart, Phys. Rev. 123, 1053 (1961); A. Martin, Phys. Rev. 129, 1462 (1963)

2. A.H. Mueller, Nucl. Phys. B 335, 115 (1990)

3. L.V. Gribov, E.M. Levin, M.G. Ryskin, Phys. Rep. 100, 1 (1983); A.L. Ayala, M.B. Gay Ducati, E. Levin, Nucl. Phys. B 493, 305 (1997); B 511, 355 (1998); Y.U. Kovchegov, Phys Rev. D 60, 034008 (1999); I. Balitsky, Nucl. Phys. B 463, 99 (1996)

4. L. Mc Lerran, R. Venugopalan, Phys. Rev. D 49, 2233, 3352 (1994); 50, 2225 (1994); 53, 458 (1996); E. Iancu, A. Leonidov, L. Mc Lerran, Phys. Lett. B 510, 133 (2001); Nucl. Phys. A 692, 583 (2001); E. Ferreiro, E. Iancu, A. Leonidov, L. McLerran, [hep-ph/0109115], to be published in Nucl. Phys. A; J. Jalilian-Marian et al. Phys. Rev. D 59, 034007 (1999); N. Armesto, M.A. Braun, Eur. Phys. J. C 20, 517 (2001); [hep-ph/0107114]; A. Kovner, U.A. Wiedemann, Phys. Rev. D 64, 114002 (2001)

5. A. Capella, E.G. Ferreiro, A.B. Kaidalov, C.A. Salgado, Nucl. Phys. B 593, 336 (2001)

6. A. Capella, E.G. Ferreiro, A.B. Kaidalov, C.A. Salgado, Phys. Rev. D 63, 054010 (2001)

7. V.N. Gribov, ZhETF 57, 654 (1967)

8. V.A. Abramovsky, V.N. Gribov, O.V. Kancheli, Sov. J. Nucl. Phys. 18, 308 (1974)
9. K. Golec-Biernat, M. Wüsthoff, Phys. Rev. D 59, 014017 (1999); D 60, 114023 (1999)

10. A.L. Ayala, M.B. Gay Ducati, V.P. Gonçalves, Phys. Rev. D 59, 054010 (1999)

11. M.B. Gay Ducati, V.P. Gonçalves, Phys. Lett. B 466, 375 (1999)

12. A.L. Ayala, M.B. Gay Ducati, E.M. Levin, Phys. Lett. B 388, 188 (1996)

13. M. McDermott, L. Frankfurt, V. Guzey, M. Strikman, Eur. Phys. J. C 16, 641 (2000); N. Armesto, C.A. Salgado, Phys. Lett. B 520, 124 (2001); E. Gotsman et al., J. Phys. G 27, 2297 (2001)

14. K.J. Eskola, K. Kajantie, P.V. Ruuskanen, K. Tuominen, Nucl. Phys. B 570, 379 (2000); K.J. Eskola, K. Kajantie, K. Tuominen, Phys. Lett. B 497, 39 (2001); D. Kharzeev, M. Nardi, Phys. Lett. B 507, 121 (2001); D. Kharzeev, E. Levin, [nucl-th/0108006]; A. Capella, D. Sousa, Phys. Lett. B 511, 185 (2001); A. Krasnitz, R. Venugopalan, Phys. Rev. Lett. 86, 1717 (2001); 84, 4309 (2000); A. Accardi, Phys. Rev. C 64, 064905 (2001)

15. A. Capella, A.B. Kaidalov, C.A. Salgado, in preparation

16. M.B. Gay Ducati, V.P.B. Gonçalves, M.V.T. Machado, Phys. Lett. B 506, 52 (2001)

17. M.B. Gay Ducati, V.P.B. Gonçalves, M.V.T. Machado, Nucl. Phys. A 697, 767 (2002)

18. K.A. Ter-Martirosyan, Nucl. Phys. B 36, 566 (1972)

19. A. Donnachie, P.V. Landshoff, Phys. Lett. B 296, 227 (1992); B 437, 408 (1998)

20. N. Nikolaev, B.G. Zakharov, Z. Phys. C 49, 607 (1990); N. Nikolaev, E. Predazzi, B.G. Zakharov, Phys. Lett. B 326, 161 (1994); J.R. Forshaw, D.A. Ross, Quantum chromodynamics and the pomeron (Cambridge University Press, 1997)

21. A. Capella et al., Phys. Lett. B 343, 403 (1995); A. Capella et al., Phys. Rev. D 53, 2309 (1996)

22. J. Bartels, M. Wüsthoff, J. Phys. G Nucl. Part. Phys. 22, 929 (1996); J. Bartels et al., Eur. Phys. J. C 7, 443 (1999)

23. ZEUS Collaboration, Eur. Phys. J. C 6, 67 (1999); ZEUS Collaboration, Measurement of the diffractive cross section at $Q^{2}<1 \mathrm{GeV}^{2}$ at HERA, in Proceedings ICHEP2000, Osaka, Japan (2000) [plenary session 12, paper 435]

24. H1 Collaboration, Measurement of the Diffractive Structure Function $F_{2}^{D(3)}\left(x_{\mathrm{P}}, \beta, Q^{2}\right)$ at HERA, in Proceedings Conference EPS2001 Budapest, July 12 (2001); and in Proceedings Conference Lepton-Photon2001, July 23 (2001) 